\title{
Cell Interactions in the Differentiation of a Melanotic Tumor in Drosophila
}

\author{
ROSE M. RIZKI and T. M. RIZKI \\ Division of Biological Sciences, University of Michigan, \\ Ann Arbor, Michigan 48109, USA
}

The cellular events in the formation of melanotic tumors in the $t u$ - $W$ mutant larva of Drosophila melanogaster are described. The first step is the differentiation of spherical hemocytes to flattened cells, the lamellocyte variants. Subsequently, the surface of the caudal fat body undergoes changes to which the hemocytes respond by forming cellular capsules. The hemocytes utilize two mechanisms in this process: (1) phagocytosis of small particulate materials escaping from the adipose cells, (2) adhesion to form a multilayered wall of lamellocytes.

Differentiating hemocytes in the vicinity of the tumor-forming site extrude membrane-bound vesicles that tend to adhere to the hemocyte surfaces. These vesicles are trapped between the lamellocytes as they pile in layers to form the capsule wall. It is suggested that the vesicles play a role in lamellocyte-to-lamellocyte adhesion during the initial stages of hemocyte aggregation at the tumorforming site.

\section{Introduction}

The first case of a hereditary melanotic tumor in Drosophila melanogaster was discovered in 1915 [1]. Since then many mutant strains showing aberrant internal black masses have been isolated, and the term 'melanotic tumor' has been used in a generic sense to identify this phenotype associated with the expression of a mutant gene. Whether this phenotypic similarity among melanotic tumor mutants is superficial or these non-allelic mutant genes block different steps in a sequential chain of molecular and cellular events has not been explored. Such an analysis requires information on the cell types involved in melanotic tumor formation, the changes occurring in these cell types prior to tumorigenesis, and the interactions between these cells as tumorigenesis progresses.

The cellular components of the melanotic tumors in the tumor-W $(t u-W)$ mutant have been examined in greater detail than melanotic tumors in any other mu-

In appreciation of his encouragement in the initiation of this research and three decades of friendship, we respectfully dedicate this article to our esteemed colleague, Professor Donald F. Poulson tant. The phenogenetics of this mutant isolated by Gowen was described by Wilson et al. [2] who concluded that a region on the second chromosome is the cause of the abnormality. Sparrow [3] recently suggested that the $t u-W$ phenotype is due to two different mutant genes, but our data indicate that $t u-W$ is a single gene in the second linkage group (manuscript in preparation). Therefore, the eventual goal of determining whether the initiation of the $t u-W$ cellular syndrome of melanotic tumors results from a loss of gene regulation and/or lack of a single gene product is still reasonable. The large melanotic masses in $t u$ - $W$ larvae result from an interaction between hemocytes and the caudal adipose tissue [4]. Cell proliferation is not involved in their formation. The present report examines the details of the cellular events in the differentiation of this mutant phenotype.

\section{Methods}

Larvae from the $t u-W(2-63.8 \pm)$ and the Ore- $R$ wild-type (nontumorous) strains were collected within $2 \mathrm{~h}$ after hatching and raised on cream of wheat-molasses medium seeded with Fleischmann's 
yeast at $24.5^{\circ} \mathrm{C}$. Under these conditions the second larval molt occurs at approximately $48 \mathrm{~h}$ and pupariation at about $96 \mathrm{~h}$. Larvae of appropriate ages were fixed in buffered formaldehyde fixative followed by post-fixation in osmium fixative for electron microscopy. Specimens for scanning electron microscopy (SEM) were dehydrated through a graded series of alcohols, amyl acetate, and processed through critical point drying. They were mounted on aluminum stubs, sputter-coated with gold, and examined in a JEOL JSM-U3 SEM. The caudal fat bodies for transmission electron microscopy (TEM) were dehydrated in alcohol, transferred to propylene oxide, and embedded in epon. Thin sections were stained with uranyl acetate and Reynold's lead citrate, and examined in a Philips 300 TEM.

\section{Results}

Classification of the larval hemocytes of $D$. melanogaster is based on studies of hemolymph samples by phase microscopy [5]. There are two types of spherical cells: those with large crystal-like inclusions are referred to as crystal cells, and cells lacking these cytoplasmic granules are the plasmatocytes. Two variants of the plasmatocyte category occur: cells with filamentous surface projections and membranous folds are designated podocytes while the flattened, disk-like variant is called a lamellocyte [6].

Both plasmatocytes and podocytes are found within the body cavities of $O r e-R$ and $t u-W$ larvae examined in the SEM (Figs. 1-4). Plasmatocytes in Ore-R show a relatively smooth surface dotted with small surface blebs, but the surfaces of $t u$ - $W$ hemocytes appear more active generally with a variety of membranous folds and filaments as well as large and small knobs. The morphologic characteristics of the lamellocyte variant are best demonstrated by examining two views of the same cell from an early third instar $t u-W$ larva (Figs. 8 and 9). There remains no doubt as to the shape of these cells which have often been reported as spindle shaped in studies of Drosophila melanotic tumors employing sectioned material. The fully differentiated cells are extremely flattened. Although prominent filaments have not been seen on the upper and lower surfaces of these cells, the borders may retain short filaments (Fig. 10). Small surface knobs or blebs, however, are present on both sides of the flattened surface. The thickness of the lamella at the border of the lamellocyte in Figure 10 is about $0.1 \mu$ and agrees with measurements on sectioned lamellocytes under TEM. The boat-shaped lateral profile is characteristic of lamellocytes resting on various surfaces of the internal organs in the fixed preparations. That these contours are not artifacts of fixation is suggested by the fact that circulating lamellocytes in the dorsal vessel of $t u-W$ larvae examined with the light microscope show the same profile.

Hemocytes in various stages of transition to the lamellocyte form have also been observed in the SEM (Figs. 5-7). These cells have globules of various sizes adhering to their surfaces by membranous attachments and filamentous borders. Globules as large as $2.5 \mu$ in diameter have been observed. The association between the globules and the differentiating cells suggests that the morphologic transition from a spherical to flattened shape is accompanied by extrusion of intracellular fluids. Small knobs on the transforming hemocytes measure $0.1-0.2 \mu$ and are in the same size range as knobs on Ore- $R$ plasmatocytes of the same age larvae.

Melanotic tumor formation in $t u-W$ is limited to the caudal region of the larval fat body. The fat body is covered by a basement membrane and the surfaces of Ore- $R$ and $t u-W$ adipose tissue are alike when examined in the SEM (Fig. 11). The first sign of deviation from normality at the surface of the prospective tumor-forming site appears at approximately $60 \mathrm{~h}$ with a rounding up of the caudal fat body cells (Fig. 12). Globular and membranous materials appear at the intercellular boundaries of the adipose cells. At this stage an occasional hemocyte can be seen resting on the surface of the adipose tissue near the accumulations. In the Ore- $R$ larval fat bodies no hemocytes were encountered adhering to the tissue surfaces. Either the adherence of hemocytes to normal fat body is rare or absent.

Thin sections through the tumor-forming site of $t u$ $W$ larvae at $60 \mathrm{~h}$ reveal that the hemocytes are actively engulfing materials oozing from the surfaces of the adipose cells (Fig. 15). Basement membrane is broken or absent in some regions of the adipose cell surfaces. A section through undisturbed basement membrane of $t u$ $W$ fat body is included to illustrate how this surface material between the tracheal cell and the adipose cell inhibits direct contact between the surfaces of the two cell types (Fig. 16). Ruthenium red treated material shows high electron density at the surface of the basement membrane and less dense material with disoriented fine fibrils underneath. When the basement membrane of the caudal fat body undergoes dissolution, the tracheal cells become decemented from the adipose tissue surface and some of the globular and membranous material seen by SEM at the intercellular boundaries must include this material (compare SEM Fig. 12 and TEM Fig. 15). At higher magnification the absence of basement membrane over the surface of adipose cells can be seen more clearly (Fig. 21). The phagocytic activity by the hemocytes resting on the adipose cell surfaces is also evident. The material being engulfed by the hemocyte in 

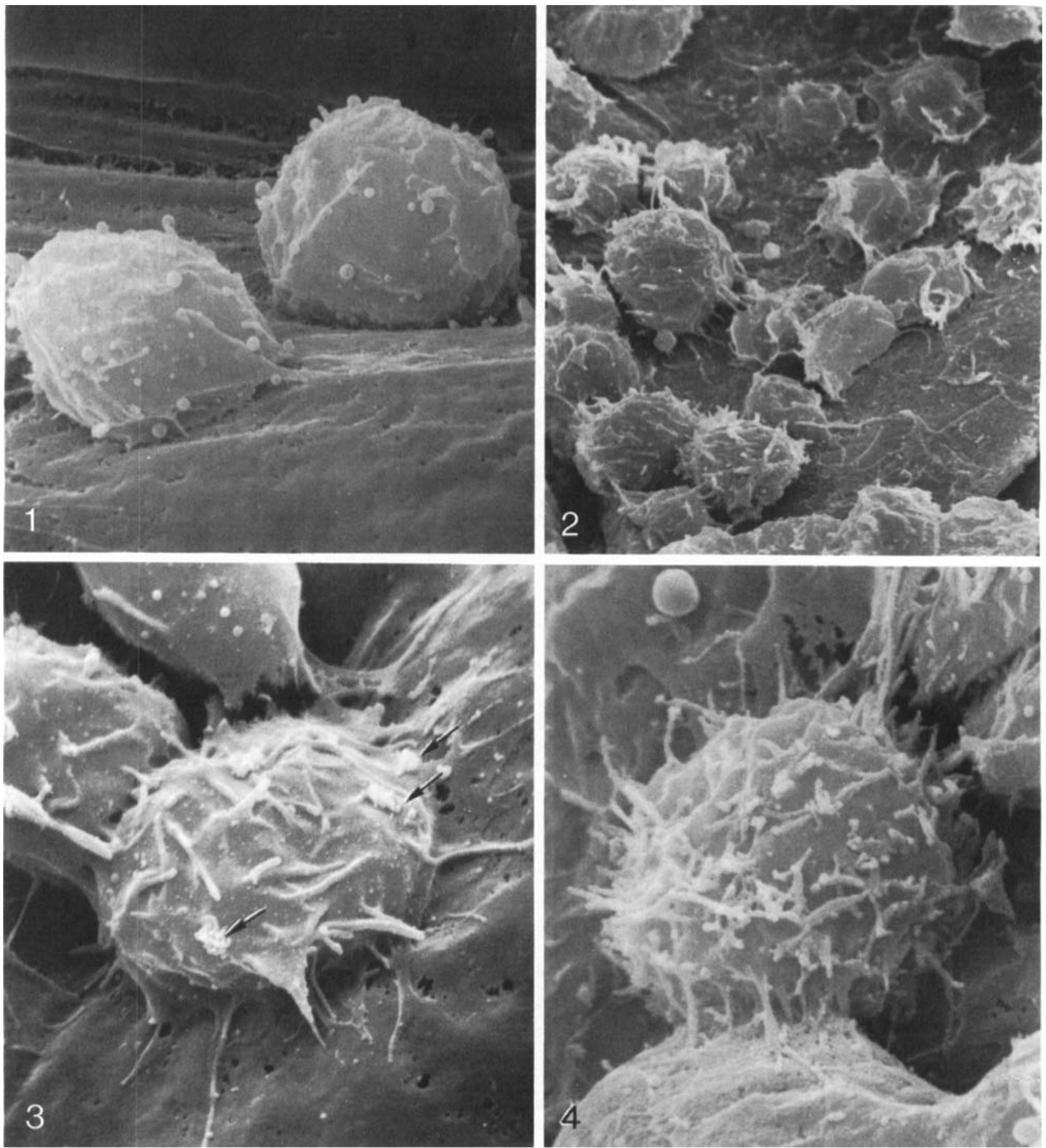

Fig. 1. Two Ore-R plasmatocytes resting on the surface of segmental muscles show knobs which vary from $0.1-0.2 \mu$ in diameter. Larval age, 86 h. $\times 10,000$

Fig. 2. A group of hemocytes in the body of a $t u-W$ larva. The hemocytes are pleomorphic; their surfaces show numerous filaments, membranous folds, and bubbles. Bubbles are also free in the surrounding region. Larval age, $80 \mathrm{~h} . \times 3,000$

Fig. 3. An Ore- $R$ podocyte resting on the ventral surface of the caudal hemocoel (anal organ region). There is a regional differentiation of the hemocyte surface with respect to the morphology of the filamentous extensions; those near the substratum are membranous at their proximal ends and tend to spread over the surface. Those on other parts of the cell are blunt. There are a few clusters of filaments which appear as 'clenched' fingers (arrows). Larval age, $96 \mathrm{~h} . \times 10,000$

Fig. 4. A $t u-W$ podocyte in the caudal hemocoel. Note the high density of filamentous projections. Larval age, $86 \mathrm{~h} . \times 10,000$ 

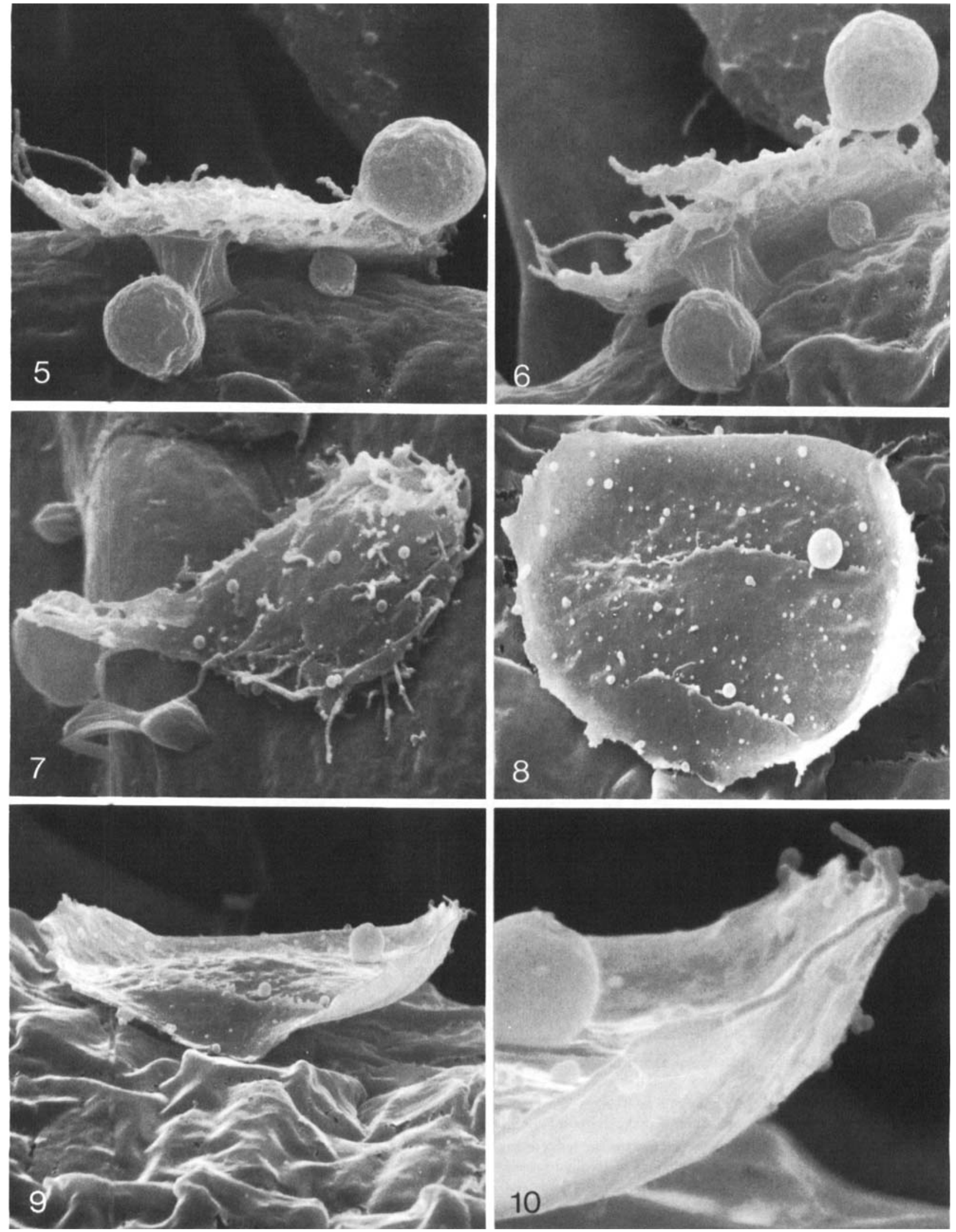

Figs. 5-10. (Legends see page 172) 

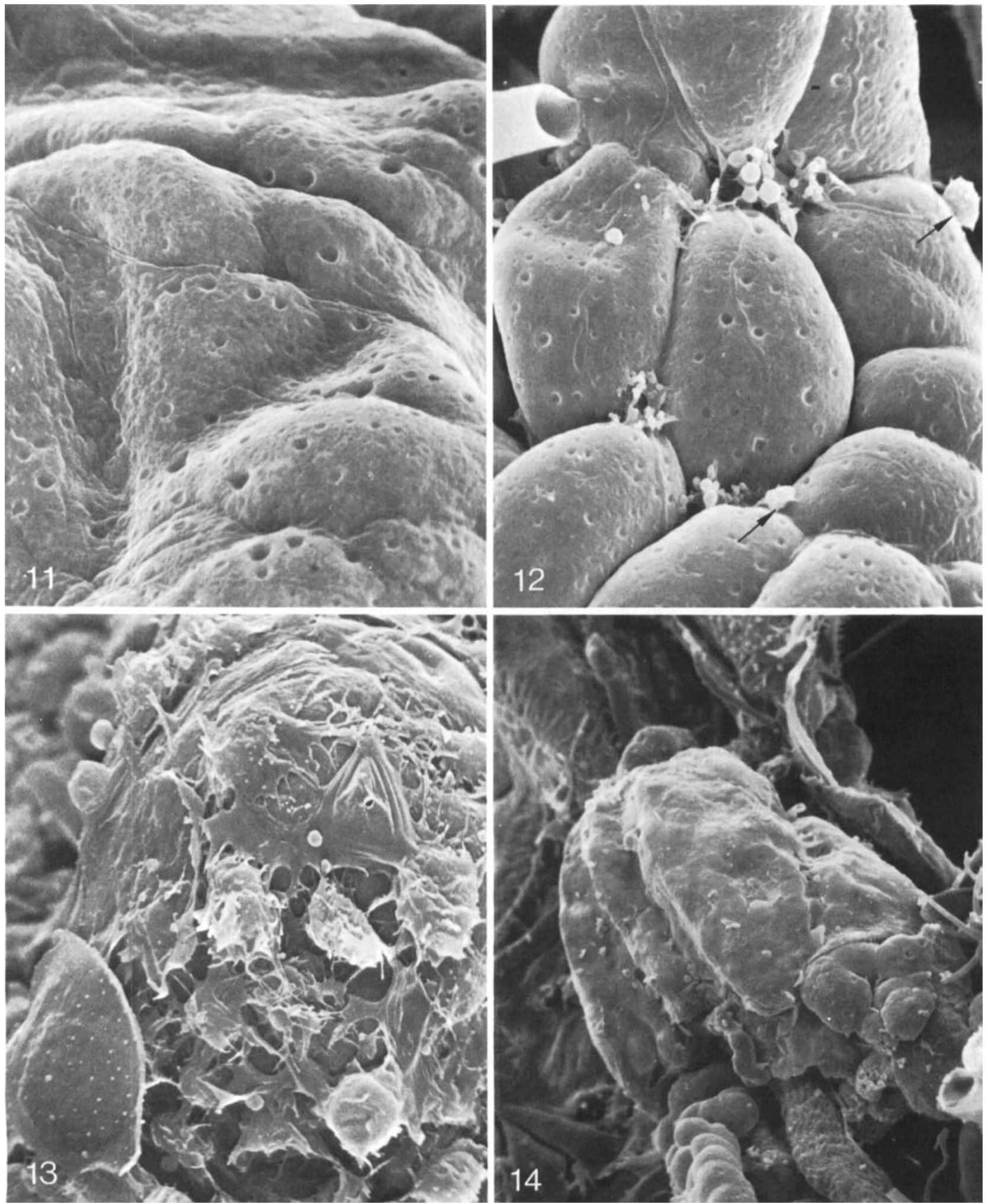

Figs. 11-14. (Legends see page 172) 
Fig. 5. A $t u-W$ podocyte differentiating into a lamellocyte. Note the membranous material with adhesive tendency for the substrate and globular extensions which vary in size from $0.7-2.3 \mu$. In this lateral profile of the cell the filamentous extensions arising from the cell surface are free as opposed to those seen in the normal podocyte in Figure 3 where they made contact with the substrate. Larval age, $60 \mathrm{~h} . \times 10,000$

Fig. 6. Same cell as Figure 5 tilted to examine the ventral surface. Note the small globule between the substratum and the cell surface, and the absence of filaments from this surface. $\times 10,000$

Fig. 7. A polar view of a nearly transformed $t u-W$ blood cell showing filaments and small knobs of material on the surface. Large globules with membranous extensions appear in the process of detachment from the cell body. As in the cell above, the filaments are more prominent at the periphery of the cell. Larval age, $86 \mathrm{~h} . \times 10,000$

Fig. 8. A polar view of a fully transformed blood cell, the lamellocyte variant. Note the presence of small knobs and globules on the cell surface; filaments are extremely reduced and can be seen only at the edges of the cell or at the borders of the pleated surface of the lamellocyte. Larval age, 72 h. $\times 4,500$

Fig. 9. Same cell as Figure 8 viewed from the side. In the lateral profile the discoid cell appears boat-shaped

Fig. 10. Part of the frame in Figure 9 magnified to resolve surface details of the lamellocyte. Small protrusions can be seen on both surfaces; fine filaments extending from the edge of the cell body can also be seen. The large globule sitting on the upper surface measured $1.5 \mu$ in diameter; the thickness of the cell body is between $0.15-0.2 \mu$. It should be noted that the substrate is the basement membrane of an internal organ and the two surfaces, the cell and substratum, are in repulsion. A similar behavior of the surface in profile was observed with lamellocytes resting on a glass surface [6]. $\times 16,000$

Fig. 11. The caudal fat body surface of an Ore- $R$ larva. The adipose cells are covered by basement membrane and the major prominences of the surface contour correspond to the underlying adipose cells. The pits on the surfaces are also covered by membrane. Larval age, $64 \mathrm{~h}$. $\times 1,500$

Fig. 12. The caudal fat body of $t u-W$ illustrating the early changes in the topography. Each adipose cell is rounded up; the contours of the tracheoles overlying the cells are clearly discernible. The basement membrane shows interruptions at the points where three or four cells are coming together, and membranous fragments and globular materials are seen at these intercellular spaces. Hemocytes (arrows). Larval age, $64 \mathrm{~h}$. $\times 1,500$

Fig. 13. A region of $t u-W$ caudal fat body in the process of capsule formation by hemocytes. A lamellocyte can be seen in the lower left. Some of the blood cells are in transition form an ellipsoidal to flattened form; the thinness of these spreading cells is apparent. Note also the membranous extensions and the apparent stickiness of the hemocytes. Large and small globules are scattered over the surface. At the higher magnification at least two or more layers of flattened cells can be resolved in this specimen. Larval age, $96 \mathrm{~h} . \times 3,000$

Fig. 14. A fully formed melanotic tumor. The adipose cells of the caudal fat body are completely covered by encapsulating hemocytes. Larval age, 96 h. $\times 200$

Fig. 15. A phagocytic hemocyte on the surface of the fat body where three adipose cells are undergoing loss of basement membrane. The alveolar contours appear to arise from the lifting of the outer surface (os) of the basement membrane (b). Projections (p) arising from the hemocyte are extending between the intercellular space and also apposing the surface of the tracheoles $(t)$. Larval age, $60 \mathrm{~h} . \times 7,400$

Fig. 16. Section showing the surface of an unaffected adipose cell in a $t u-W$ larva. Note how the basement membrane material (b) separates the tracheal cell $(t)$ from the surface of the adipose cell. In this ruthenium red preparation the surface of the basement membrane shows greater electron density than the inner matrix. Lipid globule $(L)$ within the adipose cell. Larval age, $86 \mathrm{~h} . \times 18,900$

Fig. 17. Two hemocytes in the process of engulfing extruded 'protein globules' $(g)$ from $t u$ - $W$ adipose cells $(f)$. Note one globule between the two affected adipose cells, one within the cell body of a hemocyte, and one adhering to the hemocyte surface probably in the process of being engulfed. Ruthenium red stain. Larval age, $86 \mathrm{~h} . \times 24,000$

Fig. 18. Section through a group of transforming hemocytes in a $t u$-W larva showing the extrusion of surface vesicles. The one from the end of a surface filament is $0.25 \mu$ in diameter; the smallest vesicle profile measures $0.08 \mu$ in diameter. Larval age, $60 \mathrm{~h} . \times 60,000$

Fig. 19. Section through lamellocytes near the tumor-forming site. Note the free round vesicles and the collapsed vesicles adhering to the surfaces of the hemocytes. Larval age, $86 \mathrm{~h} . \times 24,900$

Fig. 20. The wall of the tumor formed by the layer of lamellocytes. The electron dense regions (arrows) are remnants of the flattened vesicles sandwiched between the surfaces of the lamellocytes. Larval age, $96 \mathrm{~h} . \times 49,000$ 

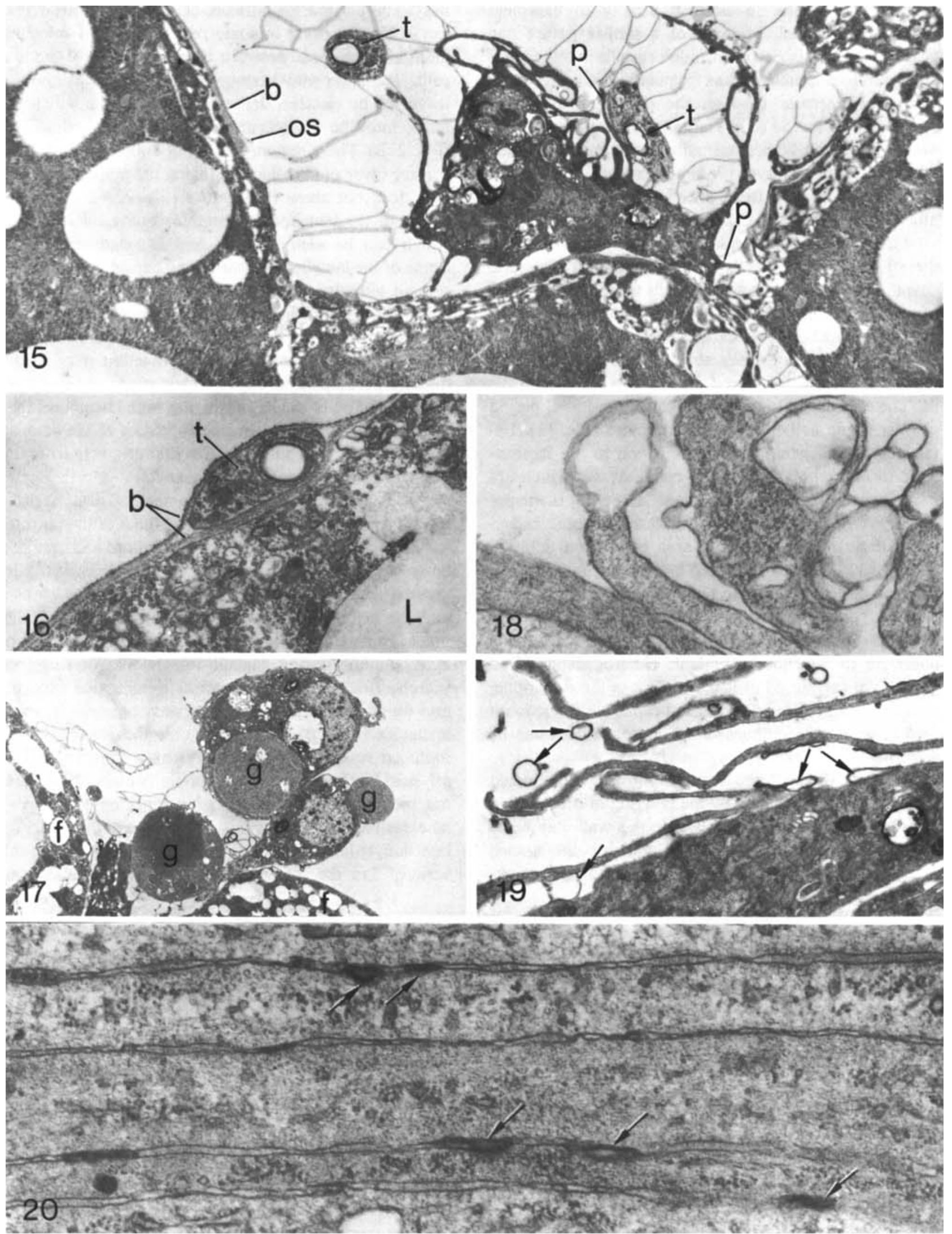

Figs. 15-20. (Legends see page 172) 
Figure 21 is similar in electron density to basement membrane material. Material of a similar nature can also be seen within one of the adipose cells in this photograph, and such material has frequently been seen in the intercellular spaces between the dissociating adipose cells. In older larvae $(80 \mathrm{~h}$ ) large globules similar to the protein inclusion bodies normally found in the fat body of mid third instar larvae [7, 8] are also lost from the $t u$ $W$ adipose cells, and these are phagocytized by the hemocytes as well (Fig. 17).

Mitochondria and endoplasmic reticulum (ER) in the affected fat body cells show no apparent structural deviations from normal adipose cells when the first signs of surface changes appear. Synthetic activity as viewed by intracisternal accumulations is also pronounced in some of the tumorous cells at this stage (study in progress).

The encapsulation process follows the initial period of phagocytic activity by the hemocytes (Fig. 13). Lamellocytes and hemocytes in transition to the lamellocytic form land on the adipose cell surfaces which apparently lack basement membrane. Layering of hemocyte upon hemocyte continues until a laminated capsular wall surrounds the entire area of afflicted adipose cells. There does not appear to be a distinction between the type of cell adhering to the outermost surface of the capsule; some cells are fully differentiated lamellocytes and some cells are in the stage of flattening from the podocyte to lamellocyte variant. Heterogeneous sized globules are scattered over the surface of the developing capsule. A fully formed melanized capsule has a smooth surface where the outlines of the flattened cells can be discerned (Fig. 14).

Sections through the capsule show the laminated nature of the wall formed by the layering of the lamellocytes (Fig. 22). Lamellocytes within this wall may be as thin as $0.1 \mu$, but the regions containing the nuclei, which are somewhat thicker, are about $1.5 \mu$, in thick- ness. The plasma membranes of apposing lamellocytes are in close contact in some regions whereas other regions show a space between the membranes of the two cells. The latter may be long or short stretches and are traversed by electron dense fibrillar material which extends into the cytoplasm of the cells on either side (Fig. 22B). These regions are about $200 \AA$ in width. The surface layer of lamellocytes facing the adipose cell surface does not show this feature (Fig. 22A). The cytoplasm of the lamellocytes contains many microtubules which can be seen in cross and longitudinal section. Some of the lamellocytes within the capsule wall contain protein globules similar to those found in the adipose cells. Presumably these inclusions represent phagocytic materials within the hemocytes. If this is so, then a cell engaged in the initial phagocytic reaction may subsequently participate in capsule formation.

In addition to the large globules in the hemocoel that are extruded from the disturbed surfaces of the adipose cells, there are also small vesicles that originate from the surfaces of the hemocytes. The vesicles seen in Figure 18 measure $0.08-0.25 \mu$ in diameter. These vesicles have a tendency to adhere to the surfaces of the plasmatocytes at the time of active encapsulation, and can also be seen flattening and spreading over the hemocyte surfaces (Fig. 19). This adhesiveness between the vesicles and the hemocyte surfaces suggests that the vesicular materials play a role in the initial lamellocyte-to-lamellocyte binding during capsule formation. We therefore searched for comparable material in laminated capsules and found such entrapments between the cells in some instances (Fig. 20). It appears that the electron-dense flattened vesicles of various sizes may form regions of physical hindrance preventing direct contact of the plasma membranes of two apposing lamellocyte surfaces whereas regions lacking this material may form a close lamellocyte-lamellocyte contact. The lipid bilayers of the vesicles and the lamellocyte plasma membranes may

Fig. 21. A hemocyte actively engaged in phagocytosis at the junction of two $t u$ - $W$ adipose cells. This section of caudal fat body corresponds to the region illustrated in Figure 12 where membranous globules are accumulated. Note that the basement membrane of the adipose tissue is disrupted at the level of a projection arising from the hemocyte $(p)$ and the amorphous material engulfed by the hemocyte has an electron density similar to basement membrane. This material $(a)$ is seen within and between the adipose cells, in the hemocyte, and between the projections extending from the hemocyte. $L$, lipid globules; $b$, basement membrane; $t$, tracheal cell. Larval age, $60 \mathrm{~h} . \times 20,900$

Fig. 22. The laminated structure of the tumor capsule resulting from close packing of the lamellocyte surfaces and the wedging developed between the membranous folds of the lamellocyte surfaces. Note the close apposition of the cell membranes and the regions of electron dense gaps (gp) of varying lengths. In some regions of the lamellocytes there is a high density of microtubules in cross section whereas in other regions they are longitudinally disposed. Larval age, $96 \mathrm{~h}$. $\times 20,900$. Inset A: An enlarged view of the first layer of lamellocytes $(l)$ against the adipose cell $(f)$ surface. Note the absence of gaps between the two surfaces. $\times 51,200$. Inset B: An enlargement of the area marked by asterisks showing the close apposition of lamellocyte surfaces and the gaps ( $g p) . \times 51,200$ 


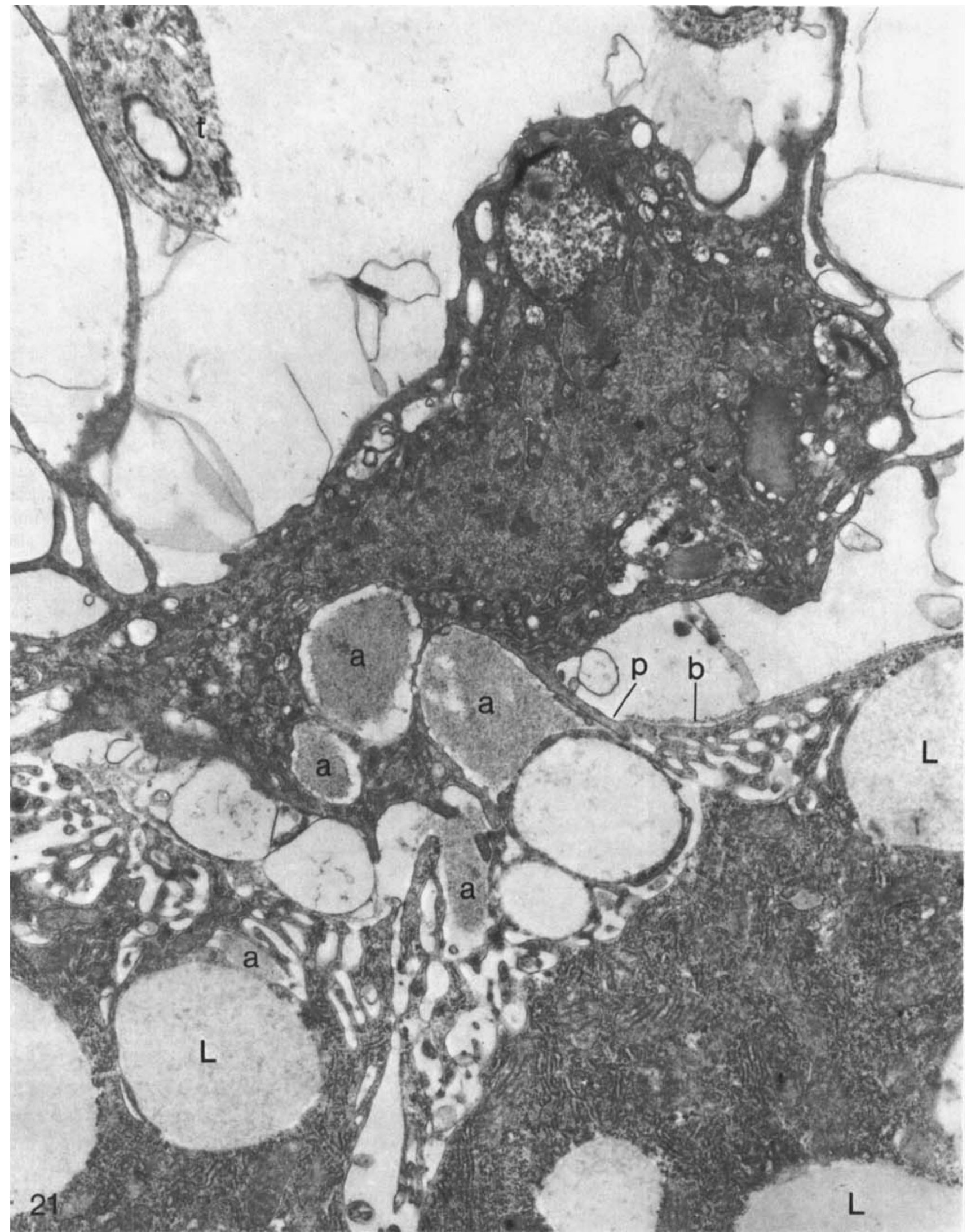

Fig. 21. (Legend see page 174) 

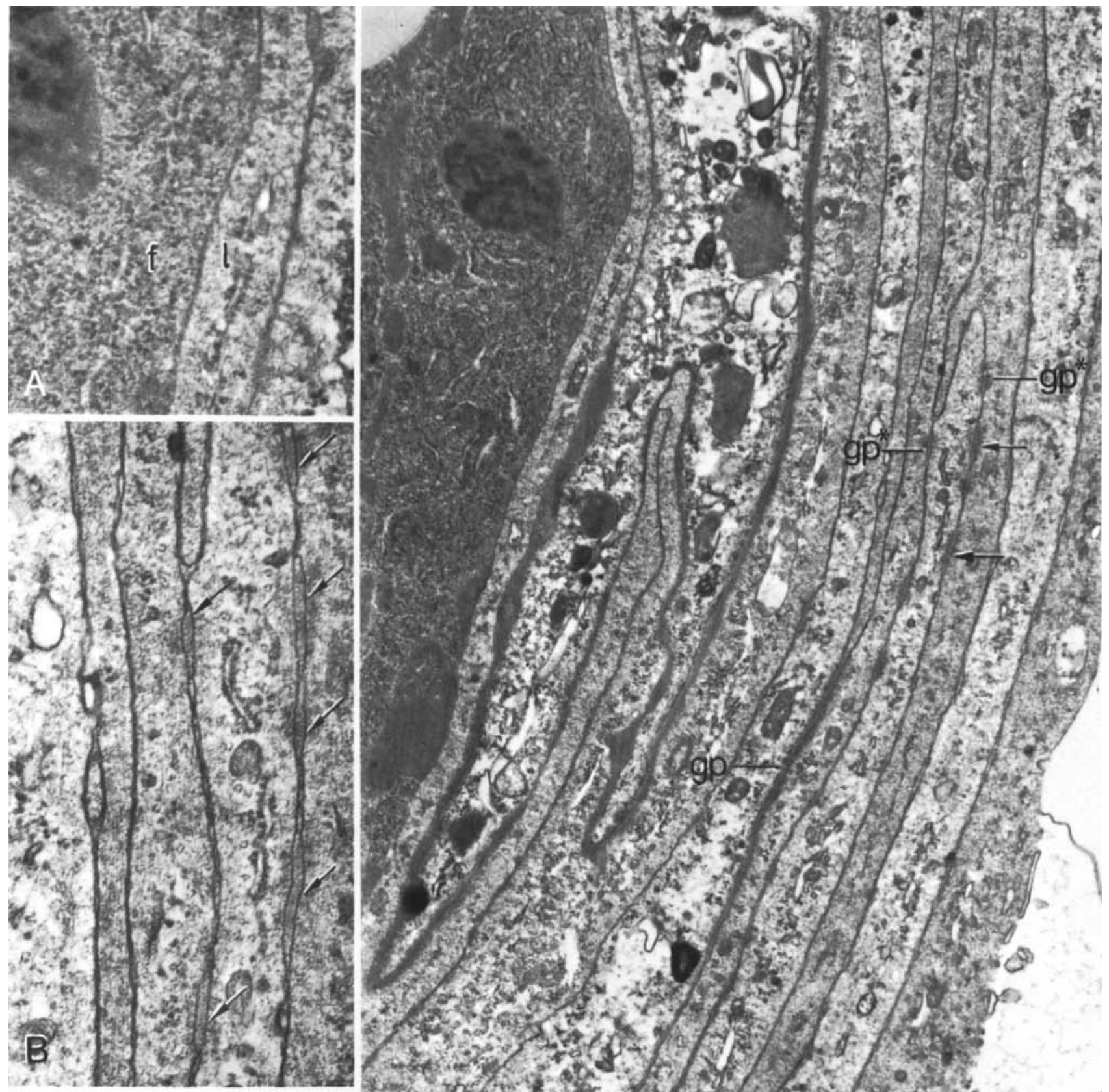
eventually amalgamate leaving a dilated region filled with electron dense material extending on either side into the cell cytoplasms as seen in Figure 22B.

\section{Discussion}

The first morphologic deviation from normality in $t u-W$ larvae is the precocious appearance of many lamellocytes in the early third instar [6]. These cells circulate throughout the hemocoel and are not associated with the caudal fat body. Only after changes are apparent in the surface of the adipose tissue does hemocyte activity at this site begin. That surface modification is a prerequisite for the encapsulation reaction has recently been tested by implantation studies using host larvae in which lamellocytes were present [9]. While the lamellocytes remained neutral to intact, unmodified fat body implants, fat bodies with mechanically injured surfaces or where the basement membrane was removed by collagenase treatment became encapsulated by lamellocytes.

Two mechanisms are utilized by the hemocytes to confine the dissemination of products from the aberrant adipose cells. The hemocytes appearing first at the caudal fat body phagocytize surface materials and debris lost from the adipose cells. Phagocytosis is the typical defense response of insect hemocytes against small foreign objects in the hemocoel [10], and this behavior of the plasmatocytes in Drosophila can be readily demonstrated by injection of bacteria (Rizki and Rizki, in preparation). The phenomenon of melanotic tumor formation in the $t u-W$ mutant is hereditary, and neither ultrastructural studies nor parabiosis of normal and tumorous larvae have demonstrated the presence of an infective agent [11]. Phagocytosis in these larvae is directed against components that are normally within the adipose cells. These 'in situ' observations on phagocytosis are of particular interest since the hemocoel is undisturbed by external stresses or experimental manipulations.

The second response of the hemocytes is encapsulation of the caudal adipose cells to produce compact capsules that later undergo melanization. What initiates the encapsulation reaction is not clear. The disturbances at the adipose cell surfaces may serve as the direct stimulus for the encapsulation response as well as the phagocytic reaction. On the other hand, it is possible that the stimulus for the encapsulation process by the lamellocytes is evoked by the activity of the phagocytes establishing first contact with the adipose cells since some of these hemocytes appear to exude material over the substrate [12].
The fact that lamellocytes appear in circulation prior to visible changes in the adipose cells suggests that the transition from the spherical to flattened hemocyte form may be independent of tumor site specific contact. It is likely that biochemical factors released into the hemolymph provide a stimulus for hemocyte differentiation. Such biochemical stimuli may be emitted from the caudal fat body cells in $t u-W$ larvae or may be due to factors external to the tumor site, such as hormonal modifications [13, 14]. These considerations do not exclude hemocyte differentiation at the tumor-forming site, however, since differentiating cells can be seen at the aggregation surface.

The role of the vesicles observed adhering to the surfaces of the lamellocytes in the vicinity of the tumorforming site is not certain. These vesicles may represent cytosol byproducts associated with the morphologic change of the spherical hemocyte to an extremely flattened shape. On the other hand, these vesicles may function in the adhesion of the lamellocytes to other lamellocytes in the initial stages of aggregation. In this regard, the difference between the adipose cell-to-lamellocyte and lamellocyte-to-lamellocyte interfaces may be significant since interference with the adhesiveness between lamellocytes is obtained by glucosamine treatment whereas the attachment of lamellocytes to adipose cells remains undisturbed [15].

Acknowledgement: This investigation was supported by Grant No. CA-16619 awarded by the National Cancer Institute, DHEW, and in part by NIH Biomedical Sciences Grant No. RR-07050.

\section{References}

1. Bridges, C. B.: Non-disjunction as proof of the chromosome theory of heredity. Genetics 1, 53 (1916)

2. Wilson, L. P., King, R. C., Lowry, J. L.: Studies on the $t u$-W strain of Drosophila melanogaster I. Phenotypic and genotypic characterization. Growth 19, 215 (1955)

3. Sparrow, J. C.: The genetics of some second chromosome melanotic tumour mutants of Drosophila melanogaster. Genet. Res. 23, 13 (1974)

4. Rizki, T. M.: Tumor formation in relation to metamorphosis in Drosophila melanogaster. J. Morphol. 100, 459 (1957)

5. Rizki, T. M.: Alterations in the haemocyte population of Droso phila melanogaster. J. Morphol. 100, 437 (1957)

6. Rizki, T. M.: Experimental analysis of hemocyte morphology in insects. Am. Zool. 2, 247 (1962)

7. Butterworth, F. M., Bodenstein, D., King, R. C.: Adipose tissue of Drosophila melanogaster I. An experimental study of larval fat body. J. Exp. Zool. 158, 141 (1965)

8. Gaudecker, B., von: Über den Formwechsel einiger Zellorganellen bei der Bildung der Reservestoffe in Fettkörper von Droso phila-Larven. Z. Zellforsch. 61, 56 (1963) 
9. Rizki, R. M., Rizki, T. M.: Tissue encapsulation in melanotic tumor mutants of Drosophila. Proc. XI Int. Colloq. Invert. Pathol. (In press)

10. Salt, G.: The cellular defence reactions of insects. Cambridge: University Press 1970

11. Rizki, T. M.: Telobiosis of normal and tumorous larvae of Drosophila melanogaster. Drosophila Inform. Serv. 32, 153 (1958)

12. Rizki, R. M., Rizki, T. M.: Basement membrane abnormalities in melanotic tumor formation of Drosophila. Experientia 30, 543 (1974)
13. Rizki, T. M.: Melanotic tumor formation in Drosophila. J. Morphol. 106, 147 (1960)

14. Madhavan, K.: Induction of melanotic pseudotumors in Drosophila melanogaster by juvenile hormone. Wilhelm Roux' Arch. Entwickl.-Mech. Org. 169, 345 (1972)

15. Rizki, T. M.: The influence of glucosamine-hydrochloride on cellular adhesiveness in Drosophila melanogaster. Exp. Cell Res. 24, 111 (1961)

Received April 1978/Accepted November 1978 